\title{
Stimulus familiarity, dogmatism, and the duration of attention',
}

\author{
BRUCE T, LECKART, OHIO UNIVERSITY \\ JOAN F. WAGNER, UNIVERSITY OF CONNECTICUT
}

Thirty-eight males and 30 females viewed each of 30 black and white photographs for as long as they wished. Half the photographs were judged by the Es to be unfamiliar to college students. The remaining photographs were judged to be familiar. After the looking task all Ss completed the Dogmatism Scale. It was predicted that the open-minded Ss would spend more time looking at the novel stimuli than the closed-minded Ss, and, that all Ss would spend more time looking at novel stimuli than familiar stimuli. The results failed to confirm the hypothesis that open-minded Ss would look longer at the novel stimuli than closed-minded Ss. However, it was found that $S$ s spent more time looking at novel stimuli than familiar stimuli regardless of the degree to which their belief systems were opened or closed. The advantage of manipulating stimulus familiarity by E-selection of the stimuli was discussed.

A number of studies have indicated that the duration of visual attention is a function of the personality characteristics of the attender (Bakan \& Leckart, 1966; Christiansen, 1961; Leckart, Keeling, \& Bakan, 1966a; McReynolds, 1963; and Zamansky, 1956, 1958). For example, Bakan and Leckart had Ss classified as extravert, ambivert, or introvert view photographs rated as extravert, ambivert, and introvert for as long as they wished. They found that extraverts looked longer at extravert pictures and that introverts looked longer at introvert pictures.

The present study had two purposes. One purpose was to investigate the influence of an open- and closed-belief system (Rokeach, 1960) on the duration of visual attention. One recent study (Day, 1966) falled to find a relationship between dogmatism and the time spent looking at a set of stimuli composed of "scenic photographs," Items from the BarronWelsh Art Scale, and line drawings. However, it is conceivable that the relationship between looking time and dogmatism depends on the type of stimuli employed. One possibility is suggested by Mikol's (1960) finding that open-minded Ss express more acceptance of unconventional or novel music than closed-minded Ss. If there is a general tendency for open-minded Ss to be more receptive to novel stimulation than closed-minded Ss, then it might be expected that open-minded Ss would spend more time looking at novel stimuli than closed-minded Ss.

The second purpose of this study was to extend the research on the relationship between stimulus familiarity and looking time by using a different technique to manipulate familiarity than has usually been em- ployed. A number of studies have shown that looking time is negatively related to stimulus familiarity (Berlyne, 1958; Cantor \& Cantor, 1964a, b; Leckart, 1966; and McReynolds, 1963), famillarity being manipulated by pre-exposing Ss to the "familiar" stimull. But, it is possible to vary familiarity without using a familiarization period by carefully constructing and/or selecting the stimuli. Berlyne (1957) constructed line drawings of animals in which novel stimuli were produced by incongruous juxtaposition of stimulus elements, e.g., the head of an elephant was drawn on the body of a dog. Berlyne's results Indicated that when Ss were allowed to obtain as many tachistoscopic exposures of the stimuli as they wished that the number of exposures obtained on incongruous figures exceeded the number of exposures on congruous figures. The present study was designed to extend this research by selecting relatively complex photographs for either high or low amounts of stimulus novelty and allowing Ss to view these stimuli in a task where they are free to look at each stimulus for as long as they wish.

Thus, it was predicted that if open- and closedminded Ss were allowed to view a series of novel and familiar stimuli that (a) open-minded Ss would spend more time looking at the novel stimuli than the closed-minded Ss, and (b) all Ss would spend more time looking at novel stimuli than familiar stimull regardless of the degree to which their belief systems were opened or closed.

\section{Subjects}

METHOD

The Ss were 38 male and 30 female volunteers from an introductory psychology course.

\section{Apparatus}

The stimull were 30 black and white photographs selected from the covers of Science magazine and reproduced on $35 \mathrm{~mm}$ slides. Half of the photographs were pictures of landscapes, cloud formations, birds, and other objects judged by the Es to be familiar to college students. The remaining photographs were pictures of unusual microscopic organisms, crystal structures, and primitive tools judged by the Es to be unfamiliar or novel to untrained observers. The Es attempted to select the stimuli so that the familiar and unfamiliar stimuli would be approximately equal in complexity. The stimuli were projected on a white lenticular screen by a Kodak Carousel 800 projec- 
Table 1.

Mean looking times at the novel and familiar stimuli for the four groups of Ss

\begin{tabular}{lcc} 
Groups & \multicolumn{2}{c}{ Stimuli } \\
High Dogmatic & 8.3 & Familiar \\
Medium-High Dogmatic & 9.8 & 6.9 \\
Medium-Low Dogmatic & 8.7 & 7.2 \\
Low Dogmatic & 9.3 & 6.6 \\
\hline
\end{tabular}

tor placed $16 \mathrm{ft}$. from the screen. Ss sat $12 \mathrm{ft}$. from and facing the screen on which a 32 in. $x 48$ in. image was projected. The stimuli were changed with a manual switch provided the Ss. Looking times were automatically recorded to the nearest .1 sec. with an Esterline-Angus event recorder. Neither the recorder nor the supporting electronics could be seen or heard by the Ss.

\section{Procedure}

The Ss were asked to view the pictures one at a time, looking at each photograph for as long as they wished. They were told that they would not be asked to remember any of the stimuli or anything about them.

Before viewing the photographs, Ss were told there would be two additional parts to the experiment. The first of these two parts was a rating procedure designed to provide Ss with closure on the looking task. Immediately after the rating task Ss were taken into an adjoining room where the Dogmatism Scale was administered. To encourage honest responding on the Dogmatism Scale, the $E$ did not obtain S's name at any time before, during, or after the experiment.

\section{RESULTS}

On the basis of the Dogmatism Scale scores, the Ss were divided into four groups of 17 Ss each: High Dogmatic, $\bar{X}=+12.18$, range $=+40$ to -1 ; MediumHigh Dogmatic, $\bar{X}=-10.35$, range $=-3$ to -18 ; MediumLow Dogmatic, $\overline{\mathrm{X}}=-26.00$, range $=-19$ to -34 ; Low Dogmatic, $\bar{X}=-47.24$, range $=-35$ to -115 . For each $S$ the total time spent looking at the novel and the familiar stimuli was computed. The mean looking times at novel and familiar stimuli for the four groups are presented in Table 1. An analysis of variance reveals that there is no significant difference between groups $(F=.175, d f=3 / 64, p>.05)$ and no significant Group by Familiarity interaction ( $F=$ $.970, \mathrm{df}=3 / 64, \mathrm{p}>.05)$. However, the familiarity effect was significant ( $F=44.588, d f=1 / 64, p<.01$ ). In addition, the calculation of a correlation coefficient failed to reveal a significant relationship between Dogmatism scores and looking time $(r=-.08, t=.652, p>.05)$.

\section{DISCUSSION}

The results falled to confirm the hypothesis that open-minded Ss would be more attentive to novel stimuli than closed-minded Ss. No evidence was found indicating that the open-minded Ss spent more time attending to the novel stimuli than the closed-minded Ss. This suggests that the finding that open-minded Ss are more accepting of novel auditory stimuli (Mikol, 1960) may not be indicative of a general tendency for open-minded Ss to be more receptive to novel stimuli than closed-minded Ss. The present findings also agree with Day's (1966) results which indicate that there is no relationship between dogmatism and the duration of attention.

The finding that Ss look longer at novel stimuli than familiar stimuli regardless of the extent to which their belief systems were open or closed was expected. One possible explanation of the longer looking times elicited by the novel stimuli is that despite the Es' attempt to control complexity the novel stimuli were more complex than the familiar stimuli. In order to evaluate this possibility, complexity ratings of the 30 stimuli were obtained from a group of 15 males and 25 females following the rationale and procedures used by Leckart and Bakan (1965). Using a 7-point rating scale with the low points defined as degrees of complexity and the high points defined as degrees of simplicity, the mean ratings obtained for the familiar and unfamiliar slides were 3.99 and 3.84 , respectively $(t=.778, d f=39, p>.05)$. Considering the relationship between complexity ratings of photographs and looking time (Bakan \& Leckart, 1965), it seems unlikely that the difference in looking times between the novel and familiar stimuli in the present study is due to the novel stimuli being more complex.

Thus, the present study demonstrates that the negative relationship between stimulus familiarity and looking time found with the pre-exposure technique is also observable when familiarity has been manipulated by the Es' selection of the stimuli rather than by familiarizing $S$ with some of the stimuli. One possible advantage of this technique would occur in situations where familiarization is neither possible nor desired. For example, Leckart, Keeling, and Bakan (1966b) have shown that familiarization trials may have effects on subsequent looking times which the E may wish to avoid.

\section{References}

Bakan, P., \& Leckart, B. T. Attention, extraversion, and stimulus-personality congruence. Percept. \& Psychophys.. 1966. 1. 355-357.

Berlyne, D. E. Conflict and information-theory variables as determinants of human perceptual curiosity. J. exp. Psychol, $1957,53,399-404$.

Berlyne, D. E. The influence of complexity and novelty in visual figures on orienting responses. J. exp. Psychol., 1958, 55, 289-296.

Cantor, J. H., \& Cantor, G. N. Observing behavior in children as a function of stimulus novelty. Child Develpm., 1964a, 35 . 119-128.

Cantor, J. H., \& Cantor, G. N. Children's observing behavior as related to amount and recency of stimulus familiarization. $J$. exp. child Psychol., 1964b, 1, 241-247. 
Christiansen, K. C. Response duration as a measure of ambivalent response tendencies. J. Pers., 1961, 29, 115-123.

Day, H. Looking time as a function of stimulus variables and individual differences. Percept. mot. Skills, 1966, 22, 423-428.

Leckart, B. T. Looking time: The effects of stimulus complexity and familiarity. Percept. \& Psychophys., 1966, 1, 142-144.

Leckart, B. T., \& Bakan, P. Complexity judgments of photographs and looking time. Percept. mot. Skills, 1965, 21, 16-18.

Leckart, B. T., Keeling, K. R., \& Bakan, P. Sex differences in the duration of visual attention. Percept. \& Psychophys., 1966a, 1, 374-376.

Leckart, B. T., Keeling, K. R., \& Bakan, P. The effect of rate of presentation on free looking time, Percept. \& Psychophys., $1966 \mathrm{~b}, 1,107-109$

McReynolds, P. Reactions to novel and familiar stimuli as a function of schizophrenic withdrawal. Percept. mot. Skill, 1963, $16,847-850$.
Mikol, B. The enjoyment of new musical systems. In M. Rokeach (Ed.), The open and closed mind. New York: Basic Books, 1960. Pp. 270-284.

Rokeach, M. The open and closed mind. New York: Basic Books, 1960.

Zamansky, H. S. A technique for assessing homosexual tendencies. J. Pers., 1956, 24, 436-448.

Zamansky, H. S. An investigation of the psychoanalytic theory of paranoid delusions. J. Pers., 1958, 26, 410-425.

\section{Notes}

1. This research was in part conducted at Michigan State University and supported by Public Health Service Fellowship 1-F1-MH21, 559-01 awarded the senior author.

2. The authors wish to thank Kenneth $\mathrm{R}$. Keeling for collecting and analyzing portions of the data.

(Accepted for publication, March 6, 1967.) 